\title{
Respiratory sensation during bronchial challenge testing with methacholine, sodium metabisulphite, and adenosine monophosphate
}

\author{
G B Marks, D H Yates, M Sist, B Ceyhan, M De Campos, D M Scott, P J Barnes
}

\begin{abstract}
Background-There is some evidence that the perception of bronchoconstriction may vary according to the nature of the provoking stimulus. The aims of this study were, firstly, to develop a method for measuring dyspnoea during induced bronchoconstriction in patients with asthma and, secondly, to apply this method to testing differences between directly and indirectly acting bronchoconstricting stimuli.

Methods - Descriptive terms suitable for quantifying respiratory discomfort due to bronchoconstriction in patients with asthma were identified in a preliminary investigation. The relation between reduction in forced expiratory volume in one second $\left(F E V_{1}\right)$ and respiratory discomfort, measured using a visual analogue scale (VAS), was then studied during challenges with three different inhaled stimuli: methacholine (MCH), sodium metabisulphite (MBS), and adenosine monophosphate (AMP). Three indices were calculated to describe the relation: the VAS value associated with a $20 \%$ fall in FEV $\left(F E V_{20} V A S\right)$; the ratio of the final VAS value to the final percentage fall in $\mathrm{FEV}_{1}$ (VAS-FEV 1 ratio); and the regression coefficient for predicting VAS from the percentage fall in $\mathrm{FEV}_{1}$ within each challenge ( $\beta$ VAS FEV ${ }_{1}$ ).
\end{abstract}

Results - "Difficulty in breathing" and "chest tightness" were selected as suitable terms for quantifying respiratory discomfort. There were no differences between the three agonists in the qualitative aspects of the respiratory sensation. In paired challenges with the same agonist the three indices were all found to be reproducible for both sensations measured. MCH induced less intense difficulty in breathing and chest tightness for a given fall in $\mathrm{FEV}_{1}$ than did AMP. There was a trend in the same direction for the comparison between $\mathrm{MCH}$ and MBS. There were no differences between AMP and MBS. FEV 20 VAS was less powerful in discriminating between agonists than the two slope indices.

Conclusions - The relation between induced reduction in $\mathrm{FEV}_{1}$ and the intensity of respiratory discomfort can be measured reliably. The indirectly acting bronchoconstricting agonists AMP and MBS induced more intense respiratory discom- fort for a given fall in $\mathrm{FEV}_{1}$ than the direct agonist MCH. This may be due to differences in unmeasured mechanical changes in the lungs or to an additional action on airway sensory nerves.

(Thorax 1996;51:793-798)

Keywords: perception, dyspnoea, bronchial provocation testing.

There is some evidence that pharmacological factors may influence the perception of breathing discomfort in asthma. Endogenous opioids reduce dyspnoea during methacholine $(\mathrm{MCH})$ challenge ${ }^{1}$ and inhalation of prostaglandin $\mathrm{E}_{2}$ increases dyspnoea during exercise in patients with asthma. ${ }^{2}$ Sont $e t$ al found that bronchoconstriction induced by the indirectly acting agonist, hypertonic saline, was perceived more intensely than bronchoconstriction induced by the direct smooth muscle agonist, $\mathrm{MCH}^{3}{ }^{3} \mathrm{On}$ the other hand, Turcotte et al did not find any difference between the effect of reductions in forced expiratory volume in one second $\left(\mathrm{FEV}_{1}\right)$ induced by allergen, exercise, or histamine. ${ }^{4}$ However, this study comprised only eight subjects and may not have had sufficient power to detect differences.

In the current study we have investigated within subject variation in the perception of bronchoconstriction induced by various stimuli. The aims of this study were, firstly, to develop a method for studying factors that influence the intensity of respiratory discomfort during induced bronchoconstriction in patients with asthma and, secondly, to apply this method to testing differences between directly and indirectly acting bronchoconstricting stimuli in their effect on respiratory sensation.

\section{Methods}

IDENTIFICATION OF DESCRIPTIVE TERMS

This preliminary qualitative investigation was conducted in adults with asthma who were having bronchial challenge tests in the course of other research protocols. Forty two challenge tests resulting in a $>20 \%$ fall in $\mathrm{FEV}_{1}$ in 32 subjects (13 women) were studied. The mean age of the subjects was 27 years, mean $\mathrm{FEV}_{1}$ at baseline was $96 \%$ predicted, and geometric mean $\mathrm{PC}_{20} \mathrm{FEV}_{1}(\mathrm{MCH})$ was 1.4 $\mathrm{mg} / \mathrm{ml}$.

The challenge agonists included $\mathrm{MCH}$ (30 challenges), MBS (eight), and AMP (four). The challenge protocol was similar to the 
experimental procedure described below except that there was no measurement of respiratory sensation during the challenge and the end of test criterion was a $20 \%$ reduction in $\mathrm{FEV}_{1}$.

Subjects were asked to complete a questionnaire at the conclusion of their challenge test before the administration of bronchodilator. The preamble to the questionnaire asked the respondent to focus on the "nature or type of discomfort not its severity". Subjects were offered six phrases as possible descriptors of the type of chest discomfort they were currently experiencing: (1) I feel breathless; (2) my chest feels constricted; (3) my breathing feels uncomfortable; (4) my chest feels tight; (5) I find it hard to breathe; (6) my asthma feels worse.

These phrases were presented in the reverse order to a random half of the subjects. They were asked to rate the appropriateness of each phrase as a description of their current sensation on a five point Likert scale ranging from very poor (1) to very good (5). They were also asked to rank each phrase from most appropriate to least appropriate.

\section{Analysis}

To confirm that all six phrases were measuring a single dimension Cronbach's alpha was estimated along with the item/total correlations. The mean appropriateness score for each phrase for each agonist was calculated. For each challenge the difference between the highest scoring and the lowest scoring phrases was calculated along with the mean of these differences over all the challenges.

\section{MEASUREMENT OF RESPIRATORY SYMPTOMS} DURING INDUCED BRONCHOCONSTRICTION

\section{Subjects}

Patients aged over 16 years with symptoms of asthma within the last 12 months and $\mathrm{PC}_{20}$ $\mathrm{FEV}_{1}(\mathrm{MCH})<16 \mathrm{mg} / \mathrm{ml}$ were eligible for inclusion if they did not have significant coexisting disease and their baseline $\mathrm{FEV}_{1}$ was $>70 \%$ predicted. None of the subjects had previously participated in research dealing with respiratory sensation, although some had been involved in the preliminary qualitative study described above and most had taken part in various previous research protocols.

\section{Study design}

Demographic and clinical data were collected at the initial visit. A standardised explanation of the aims and method of the study was given and written informed consent was obtained.

Four bronchial challenge tests were performed at weekly intervals. Each subject had one challenge with each of three agonists: methacholine chloride (MCH, Sigma Chemical Company, Poole, UK), sodium metabisulphite (MBS, Sigma), and adenosine monophosphate (AMP, Sigma). Two challenges were performed in successive weeks with one of these three agonists in each subject. The challenge order was randomised and the subjects were blinded to the agonist used.

\section{Visual analogue scale measurements}

The intensity of respiratory discomfort at each step of the challenge was measured using a visual analogue scale (VAS) (fig 1). Two sensations were measured - chest tightness and difficulty in breathing. These were selected on the basis of the preliminary investigation.

The VAS procedure was explained in a standardised manner at the beginning of each challenge. At each step subjects were presented with an A4 sheet of paper with two $10 \mathrm{~cm}$ lines - one labelled "Chest tightness" and the other labelled "Difficulty in breathing". The order of the lines was randomised between subjects but was the same for all cards seen by an individual subject. The lines were anchored at the left with "none" and at the right with "the worst I can imagine". Written instructions on each sheet requested the subject to use the lines to rate the way he/she felt in terms of chest tightness and difficulty in breathing. They were to regard the lines as representing the full range of these sensations and to rate their feelings as they were at the time.

The subjects were given a pencil and a clipboard containing 15 VAS cards each marked with the challenge step number. They were asked to mark one card before the baseline measurements and another two minutes after each dose of agonist was administered. Each card was removed from the subject's view as soon as it was completed.

The VAS lines were measured at the conclusion of each challenge by the investigator. The cards were folded to hide the challenge step number, sorted into random order, and then each line was measured to the nearest millimetre.

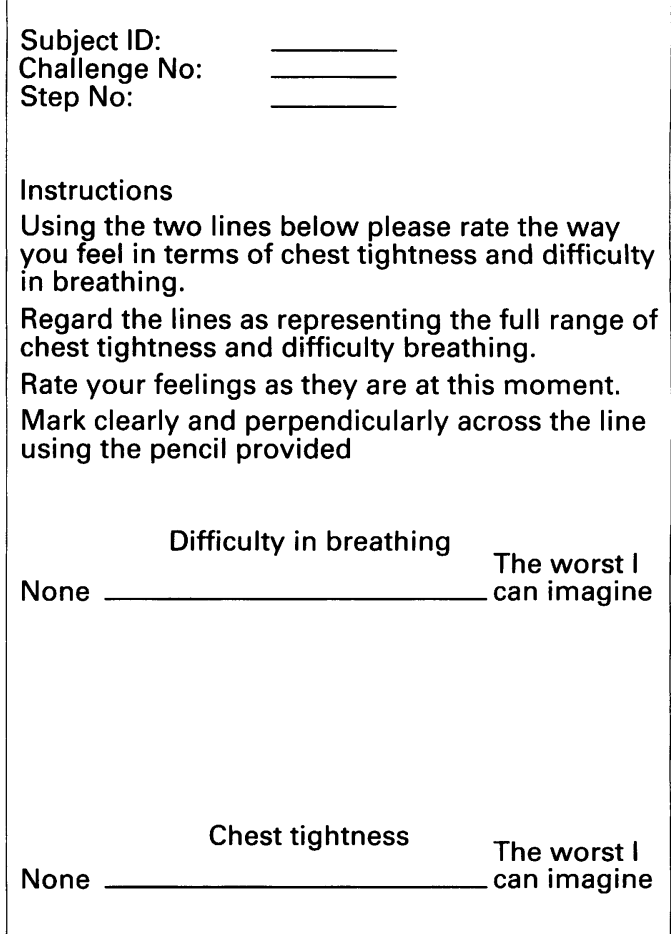

Figure 1 Visual analogue scale card used to measure the intensity of respiratory discomfort. In a random half of the subjects the order of the descriptive terms was reversed. 
Challenge procedure

Subjects were instructed to withhold short acting inhaled bronchodilators for six hours, oral and long acting inhaled bronchodilators for 24 hours, and cromoglycate and nedocromil for 12 hours prior to each challenge.

Before each challenge a VAS card was marked and then spirometric function was measured using a dry bellows spirometer (Vitalograph, Buckingham, UK). The manoeuvre was repeated until reproducible values for both $\mathrm{FEV}_{1}$ and forced vital capacity (FVC) were obtained. ${ }^{5}$ All acceptable values were recorded.

The challenge agonists were nebulised by a dosimeter (PK Morgan, Gillingham, Kent, UK) which automatically generated an aerosol at the onset of inspiration. At each dose step subjects inhaled the agonist on five successive breaths. The inhalation time was one second with a six second breath hold. Two minutes after each nebulisation subjects were asked to mark the VAS card. They then performed a single forced expiratory manoeuvre to measure $\mathrm{FEV}_{1}$. The next dose of agonist was then administered.

The first nebulisation administered in each challenge was normal saline. The post-saline $\mathrm{FEV}_{1}$ was used as the baseline for the calculation of subsequent percentage fall in $\mathrm{FEV}_{1}$. Thereafter, doubling concentrations (and hence doses) of agonists were administered. The initial and maximum concentrations administered were: methacholine, 0.06-128 $\mathrm{mg} / \mathrm{ml}$; sodium metabisulphite, $0.31-160 \mathrm{mg} /$ $\mathrm{ml}$; adenosine monophosphate, $0.39-800 \mathrm{mg} /$ $\mathrm{ml}$.

Consecutive (doubling) doses were administered until the $\mathrm{FEV}_{1}$ had fallen by $35 \%$ from the post-saline value or the last scheduled dose had been administered.

One minute after the last measurement of $\mathrm{FEV}_{1}$, and before the administration of bronchodilator, subjects were asked to complete a brief questionnaire assessing the nature of their respiratory discomfort (see below). Immediately after this they performed repeated full spirometric manoeuvres until reproducible values for $\mathrm{FEV}_{1}$ and FVC were obtained.

\section{Assessing the nature of respiratory discomfort}

At the conclusion of each challenge procedure, before the administration of bronchodilator, subjects were asked to focus on the nature of their respiratory discomfort. They were given five descriptive terms (chest tightness, breathlessness, uncomfortable breathing, wheeze, and difficulty in breathing) and asked to mark a $10 \mathrm{~cm}$ VAS line for each to indicate how good it was as a description of the type of chest discomfort they were feeling. The order of the terms was reversed in a random half of the subjects (those whose other VAS cards had "difficulty in breathing" above "chest tightness").

\section{Analysis}

The percentage fall from the post-saline values for $\mathrm{FEV}_{1}$ and the absolute values $(\mathrm{cm})$ for the VAS were used in calculations.
Three indices were calculated to describe the relation between the percentage fall in $\mathrm{FEV}_{1}$ and the VAS values: the VAS value associated with a $20 \%$ fall in $\mathrm{FEV}_{1}\left(\mathrm{FEV}_{20} \mathrm{VAS}\right)$, the ratio of the final VAS value to the final percentage fall in $\mathrm{FEV}_{1}$ (VAS-FEV 1 ratio), and the regression coefficient for predicting VAS from the percentage fall in $\mathrm{FEV}_{1}$ within each challenge ( $\beta$ VAS $\mathrm{FEV}_{1}$ ). This regression coefficient was estimated separately for each challenge without assuming a zero intercept. Goodness of fit for each linear regression was quantified as $R^{2}$. The values for the VAS-FEV ratio were log transformed for analysis.

The relative reliability of each of these indices was estimated using data from the pairs of challenges with the same agonists which each subject performed. The intraclass correlation coefficient was calculated from an analysis of variance table. ${ }^{6}$

Within subject differences between agonists in the relation between percentage fall in $\mathrm{FEV}_{1}$ from baseline and VAS values were tested by repeated measures analysis of variance. The repeated factor was the index value $\left(\mathrm{FEV}_{20}\right.$ VAS, log VAS-FEV ${ }_{1}$ ratio, and $\beta$ VAS $\mathrm{FEV}_{1}$ ) for each challenge. In addition, mean values for each of these indices for each challenge agonist were calculated across all subjects.

Between subject factors explaining differences in the above relation were tested by multiple linear regression with $\beta$ VAS $\mathrm{FEV}_{1}$ selected as the dependent variable. Adjustment for the challenge agonists was made and the following baseline characteristics were tested as covariates: age, sex, smoking status, and use of inhaled steroids. Two other covariates tested in this model were: the degree of airway hyperresponsiveness (AHR, expressed as the provoking dose of $\mathrm{MCH}$ required to induce a $20 \%$ fall in $\mathrm{FEV}_{1}$ ) and change in $\mathrm{FEV}_{1} / \mathrm{FVC}$ ratio from the baseline manoeuvre to the end of test manoeuvre.

The study was approved by the ethics committee of the Royal Brompton Hospital.

\section{Results}

IDENTIFICATION OF DESCRIPTIVE TERMS

Cronbach's alpha for the set of six descriptive phrases was 0.86 , indicating that a single dimension was measured - namely, perception of respiratory discomfort during induced bronchoconstriction. The phrase which correlated least strongly with the other items was "my asthma feels worse" (item/total correlation 0.53).

After $\mathrm{MCH}$ challenges the mean appropriateness scores for the descriptive phrases ranged from 2.73 out of 5 for "I feel breathless" to 3.47 out of 5 for "my chest feels too tight", demonstrating that all the phrases were moderate or good descriptors. However, the average difference between the most preferred and the least preferred phrase for each subject was 1.87, indicating substantial differences in individual preferences. Results were similar for questionnaires administered after AMP and MBS challenges. 
Table 1 Intraclass correlation coefficients with 95\% confidence intervals for indices of respiratory sensation relative to percentage fall in $F E V_{1}(n=23)$

\begin{tabular}{lll}
\hline & Difficulty in breathing & Chest tightness \\
\hline FEV $_{20}$ VAS & $0.83(0.64$ to 0.92$)$ & $0.68(0.39$ to 0.85$)$ \\
VAS-FEV $_{1}$ ratio (log) & $0.89(0.77$ to 0.95$)$ & $0.75(0.50$ to 0.88$)$ \\
$\beta$ VAS FEV $_{1}$ & $0.79(0.57$ to 0.90$)$ & $0.82(0.62$ to 0.92$)$ \\
\hline
\end{tabular}

$\mathrm{FEV}_{1}=$ forced expiratory volume in one second; VAS = visual analogue scale

Table 2 Mean values for indices of respiratory sensation relative to percentage fall in $F E V_{1}(n=30$ subjects)

\begin{tabular}{|c|c|c|c|c|c|c|}
\hline & \multicolumn{2}{|c|}{$F E V_{20} V A S(\mathrm{~cm})$} & \multicolumn{2}{|c|}{ VAS-FEV ratio $(\mathrm{cm} / \mathrm{l})$} & \multicolumn{2}{|c|}{$\beta V A S F E V_{1}(\mathrm{~cm} / \mathrm{l})$} \\
\hline & $D B$ & $C T$ & $D B$ & $C T$ & $D B$ & $C T$ \\
\hline AMP & 2.74 & 2.79 & 0.122 & 0.128 & 0.104 & 0.102 \\
\hline MBS & 2.52 & 2.59 & 0.149 & 0.149 & 0.107 & 0.112 \\
\hline $\mathrm{MCH}$ & 2.06 & 2.02 & 0.097 & 0.097 & 0.080 & 0.071 \\
\hline $\mathrm{p}^{1}$ & 0.05 & $<0.05$ & 0.002 & $<0.02$ & $<0.05$ & $<0.05$ \\
\hline
\end{tabular}

$\mathrm{DB}=$ difficulty in breathing; $\mathrm{CT}=$ chest tightness; $\mathrm{AMP}=$ adenosine monophosphate $\mathrm{MBS}=$

sodium metabisulphite; $\mathrm{MCH}=$ methacholine.

${ }^{1}$ Significance test for repeated measures analysis of variance (differences between agonists within subjects).

The phrases "my chest feels constricted" and "my chest feels tight" were most commonly ranked as the most appropriate descriptors (15 and 10 times, respectively). Scores for these descriptors were strongly correlated $(\mathrm{R}=$ $0.67)$.

It was concluded from this preliminary investigation that the terms "difficulty in breathing" and "chest tightness" would both be required to describe adequately the respiratory sensation experienced by people with asthma during induced bronchoconstriction.

MEASUREMENT OF SYMPTOMS DURING INDUCED BRONCHOCONSTRICTION

Thirty five patients were enrolled but five patients did not complete the study. One of these was withdrawn by the investigators because his baseline $\mathrm{FEV}_{1}$ at the second challenge was $<70 \%$ of the predicted value.

Thirty patients completed the four challenge tests. The subjects included 13 women and their mean (SD) age was 31 (8) years. The mean duration of asthma was 13 (10) years. All but one subject had at least one positive allergen skin prick test and there was one current smoker. The mean (SD) baseline $\mathrm{FEV}_{1}$ / FVC ratio was $0.80(0.08)$ and geometric mean $\mathrm{PC}_{20} \mathrm{FEV}_{1}(\mathrm{MCH})$ was $2.41 \mathrm{mg} / \mathrm{ml}$. Twenty four subjects were using short acting $\beta_{2}$ agonists and 15 were using regular inhaled steroids.

One hundred and twenty challenge tests were performed. The mean VAS scores at the end of the challenge tests were $4.1 \mathrm{~cm}$ for both "difficulty in breathing" and for "chest tightness". The mean end of test percentage fall in $\mathrm{FEV}_{1}$ was $37 \%$. The median values of $\mathrm{R}^{2}$ for the individual challenge regressions of percentage fall in $\mathrm{FEV}_{1}$ on the VAS score were 0.84 for "difficulty in breathing" and 0.83 for "chest tightness". This indicates that, on average, the relation between fall in $\mathrm{FEV}_{1}$ and the VAS scores was linear.

Reliability

In seven cases $\mathrm{FEV}_{20}$ VAS could not be calculated for at least one of the pair of repeated challenges as the final $\mathrm{FEV}_{1}$ did not fall by $20 \%$ from the post-saline values. Calculation of intraclass correlation coefficient in the remaining 23 subjects (table 1 ) revealed that all three indices were reliable. For comparison, the intraclass correlation coefficient for the provoking concentration of agonist required to induce a $20 \%$ fall in $\mathrm{FEV}_{1}\left(\mathrm{PC}_{20} \mathrm{FEV}_{1}\right)$ was 0.89 .

Differences between challenge agonists

There were significant differences among the agonists in the relation between the fall in $\mathrm{FEV}_{1}$ and VAS scores (table 2). $\mathrm{MCH}$ induced less intense difficulty in breathing and chest tightness for a given fall in $\mathrm{FEV}_{1}$ than did AMP. There was a trend in the same direction for the comparison between $\mathrm{MCH}$ and MBS, but this pairwise difference was only significant $(p<0.05)$ for the VAS-FEV 1 ratio (for chest tightness and difficulty in breathing) and for $\beta$ VAS $\mathrm{FEV}_{1}$ (for chest tightness). There were no significant differences in any of the indices between AMP and MBS.

The two scales "difficulty in breathing" and "chest tightness" differed between challenge agonists in a parallel manner ( $p>0.20$ for interaction term).

Factors explaining differences between subjects Younger subjects and those with lesser degrees of AHR had higher scores for difficulty in breathing for a given reduction in $\mathrm{FEV}_{1}$ than older subjects and those with more severe AHR $(p<0.005)$. These factors were not significant predictors of $\beta$ VAS $\mathrm{FEV}_{1}$ for chest tightness. None of the other baseline or challenge characteristics were significant independent predictors of $\beta$ VAS FEV . $_{1}$.

\section{Appropriateness of descriptive terms}

There were significant within subject differences in the perceived appropriateness of descriptive terms $(p<0.01$, repeated measures analysis of variance). The most appropriate term was "uncomfortable to breathe" (mean score $6.58 \mathrm{~cm})$. The remaining scores in order were: "difficulty in breathing" (6.19), "chest tightness" (5.85), "wheeze" (5.44), and "breathlessness" (5.11). There were no differences between the three challenge agonists in the relative appropriateness of these terms.

\section{Discussion}

Our observation that subjects found certain terms were more appropriate for describing the sensation of induced bronchoconstriction agrees with the findings of other workers who have demonstrated that patients use different words to describe respiratory sensations induced by differing stimuli and differing disease states. ${ }^{7-10}$ It was noteworthy that the qualitative questionnaire did not demonstrate any differences between the provoking stimuli in the subjects' preferred descriptive terms, which is surprising in view of anecdotal evidence that patients can distinguish among agonists (especially MBS). However, some of these sensory differences may be non-respiratory and our questionnaire focused solely on five respiratory sensory descriptions. 
A key issue in investigating the relation between change in $\mathrm{FEV}_{1}$ and the intensity of respiratory discomfort is deciding how to describe the relation. Boulet et $a l^{11}$ have used the $\mathrm{PS}_{20}$ threshold index (perception score of breathlessness at $20 \%$ fall in $\mathrm{FEV}_{1}$ which is equivalent to our $\mathrm{FEV}_{20}$ VAS. This has the disadvantage that it cannot be calculated if the $\mathrm{FEV}_{1}$ does not fall by $20 \%$ at the conclusion of the test. Furthermore, it is based on the interpolation between two points only and ignores all other data points. Sont et al analysed the VAS score corresponding to a $10 \%$ fall in $\mathrm{FEV}_{1}{ }^{3}$ This overcomes the first problem but means that sensory scores corresponding to larger falls in $\mathrm{FEV}_{1}$ are not analysed. Using the slope of the line relating $\mathrm{FEV}_{1}$ to VAS as index has the advantage that it can be calculated for all challenge tests. The advantage of the regression method ( $\beta$ VAS $F E V_{1}$ ) is that it uses all the data and does not require an assumption that the sensory score was zero at baseline.

Our main finding was that, during induced bronchoconstriction, the intensity of respiratory discomfort, for a given fall in $\mathrm{FEV}_{1}$, varies according to the agonist which induces the bronchoconstriction. In our study AMP (which induces bronchoconstriction by mast cell degranulation ${ }^{12}{ }^{13}$ and possibly also by activation of airway sensory nerves ${ }^{12}{ }^{14}$ ) and MBS (which probably acts via airway sensory nerve endings through the release of sulphur diox$\mathrm{ide}^{15}$ ) induced more severe difficulty in breathing and chest tightness for a given fall in $\mathrm{FEV}_{1}$ than did $\mathrm{MCH}$ which exerts its effect directly on airway smooth muscle. This accords with the observation of Sont $e t a l^{\beta}$ who compared another indirect agonist, hypertonic saline, with $\mathrm{MCH}$.

There are two competing explanations for the finding that indirect agonists induce more respiratory discomfort than direct agonists for a given fall in $\mathrm{FEV}_{1}$ : unmeasured mechanical changes or agonists acting on airway sensory receptors. It is possible that the indirect agonists induce mechanical changes other than those reflected in the $\mathrm{FEV}_{1}$. For example, the degree of hyperinflation induced during bronchoconstriction has been shown to explain variation between subjects in the intensity of breathlessness for a given fall in $\mathrm{FEV}_{1}$ during $\mathrm{MCH}$ challenge. ${ }^{16}$ In our study we showed that changes in the $\mathrm{FEV}_{1} / \mathrm{FVC}$ ratio (and hence changes in FVC) did not explain the observed differences between challenges. However, we did not measure inspiratory capacity or functional residual capacity and hence we cannot exclude differences between agonists in the extent of induced hyperinflation.

The alternative explanation for our finding is that AMP and MBS directly or indirectly cause increased discharge from sensory receptors within the lung. Support for this hypothesis comes from the finding of Sont et al of a correlation between changes in the index of perception and changes in the ratio of flows obtained from volume standardised maximum and partial expiratory flow volume curves (M/P ratio). ${ }^{3}$ The implication of this finding is that inflammation has a role in sensory perception.
Our study was not designed to examine variation between subjects in sensory perception of induced bronchoconstriction. However, we did examine this as a subsidiary analysis and observed, in this generally young population, that older subjects experienced less intense respiratory discomfort for a given induced fall in $\mathrm{FEV}_{1}$ than younger subjects. Connolly $e t a l$, using $\mathrm{MCH}$ induced bronchoconstriction, reached the same conclusion in a comparison between young and elderly asthmatics. ${ }^{17}$

We also observed, like Burdon et al ${ }^{18}$ but in contrast to Killian et $a l^{19}$ and Sont et al, ${ }^{3}$ that subjects with more severe AHR reported less intense respiratory discomfort for a given fall in $\mathrm{FEV}_{1}$ than those with mild AHR. This observation is difficult to interpret because it is confounded by the dose of agonist required to induce the fall in $\mathrm{FEV}_{1}$. Subjects with milder AHR will have received more agonist to induce a given fall in $\mathrm{FEV}_{1}$ than those with severe AHR. If the agonist does have a sensory effect additional to that mediated by a reduction in $\mathrm{FEV}_{1}$, then this may explain the apparent observed difference in perception.

In conclusion, we have shown that the relation between intensity of respiratory discomfort and induced reduction in $\mathrm{FEV}_{1}$ differs within subjects according to the inducing stimulus. In the clinical setting it may be important to recognise that the ability of patients to perceive airway narrowing may, in part, be a function of the factors which induced the bronchoconstriction. Understanding the basis of this variation may elucidate the mechanisms that underlie the perception of bronchoconstriction in airway diseases.

This study was funded by a grant from the National Asthma Campaign. GBM was supported during the course of this study by the Royal Australasian College of Physicians (Cottrell Fellowship) and the Asthma Foundation of New South Wales (Travelling Fellowship). He also received travel grants from the Sydney Hospitallers and the Asthma Foundation of Victoria.

We thank Mrs Miin Worsdell for assistance in conducting this
Wand study.

1 Bellofiore S, Di Maria G, Privitera S, Sapienza S, Milic-Emili J, Mistretta A. Endogenous opioids modulate the increase in ventilatory output and dyspnoea during severe acute bronchoconstriction. Am Rev Respir Dis 1990; 142:812-6.

2 Taguchi O, Kikuchi Y, Hida W, Iwase N, Okabe S, Chonan $\mathrm{T}$, et al. Prostaglandin $\mathrm{E} 2$ inhalation increases the sensation of dyspnea during exercise. Am Rev Respir Dis sensation of dysp

3 Sont J, Booms P, Bel E, Vandenbroucke J, Sterk P. The severity of breathlessness during challenges with inhaled methacholine and hypertonic saline in atopic asthmatic subjects. The relationship with deep breath-induced bronchodilation. Am $\mathcal{F}$ Respir Crit Care Med 1995;152:38-44.

4 Turcotte H, Corbeil F, Boulet L-P. Perception of breathlessness during bronchoconstriction induced by antigen, exercise, and histamine challenges. Thorax 1990;45:914-8.

5 American Thoracic Society. Standardization of spirometry: 1987 update. Am Rev Respir Dis 1987;136:1285-98.

6 Snedecor G, Cochrane W. Statistical methods. 8th edn. Iowa State University Press, 1989:242-5.

7 Simon P, Schwartzstein R, Weiss J, Lahive K, Ford V, Teghtsoonian $\mathrm{M}$, et al. Distinguishable sensations of breathlessness induced in normal volunteers. Am Rev Respir Dis 989;140:1021-7.

8 Schwartzstein R, Lilly J, Israel E, Basner R, Sparrow D, Weinberger $\mathrm{S}$, et al. Breathlessness of asthma differs from that of external resistive loading. Am Rev Respir Dis 1991; 143:A596.

9 Schwartzstein R, Carpenter E, Brughera A, Sparrow D, O'Donnell C, Banzett R. Quality of breathlessness during bronchoconstriction with different inhalational agents. $\mathrm{Am}$ Rev Respir Dis 1992;145:A630.

10 Demediuk B, Manning J, Lilly J, Fend V, Swineberger SE, Weiss JW, et al. Dissociation between dyspnea and respiratory sensation. Am Rev Respir Dis 1992;146:1222-5.

11 Boulet L-P, Leblanc P, Turcotte H. Perception scoring of induced bronchoconstriction as a index of awareness of asthma symptoms. Chest $1994 ; 105: 1430-3$. 
12 Rafferty P, Beasley R, Holgate S. The contribution of histamine to immediate bronchoconstriction provoked by inhaled allergen and adenosine 5' monophos-
phate in atopic asthma. Am Rev Respir Dis 1987;136: phate in $369-73$.

13 Bjorck T, Gustafsson L, Dahlen S. Isolated bronchi from asthmatics are hyperresponsive to adenosine, which apparently acts indirectly by liberation of leukotrienes and histamine. Am Rev Respir Dis 1992;145:1087-91.

14 Polosa R, Holgate S, Church M. Adenosine as a proinflammatory mediator in asthma. Pulmon Pharmacol 1989;2:21-6.

15 Nichol G, Nix A, Chung K, Barnes P. Characterisation of bronchoconstrictor responses to sodium metabisulphite aerosol in atopic subjects with and without asthma. Thorax 1989;44:1009-14.
16 Lougheed M, Lam M, Forkert L, Web K, O'Donnell D. Breathlessness during acute bronchoconstriction in
asthma: pathophysiologic mechanisms. Am Rev Respir Dis 1993;148:1452-9.

17 Connolly M, Crowley J, Charan N, Nielsen C, Vestal R. Reduced subjective awareness of bronchoconstriction provoked by methacholine in elderly asthmatic and normal subjects as measured on a simple awareness scale. Thorax 1992;47:410-3.

18 Burdon J, Juniper E, Killian K, Hargreave F, Campbell E. The perception of breathlessness in asthma. Am Rev Respir Dis 1982;126:825-8.

19 Killian K, Summers E, Watson R, O'Byrne P, Jones N, Campbell E. Factors contributing to dyspnoea during bronchoconstriction and exercise in asthmatic subjects. Eur Respir f 1993;6:1004-10. 